\title{
Shape evolution in the neutron-rich tungsten region
}

\author{
P. D. Stevenson, ${ }^{1}$ M. P. Brine, ${ }^{1}$ Zs. Podolyak, ${ }^{1}$ P. H. Regan, ${ }^{1}$ P. M. Walker, ${ }^{1}$ and J. Rikovska Stone ${ }^{2}$ \\ ${ }^{1}$ Department of Physics, University of Surrey, Guildford GU2 7XH, United Kingdom \\ ${ }^{2}$ Clarendon Laboratory, Oxford University, Oxford OX1 3PU, United Kingdom
}

(Received 1 September 2005; published 28 October 2005)

\begin{abstract}
Recent experimental data on the low-lying states in ${ }^{190} \mathrm{~W}$ show a change in the $E\left(4_{1}^{+}\right) / E\left(2_{1}^{+}\right)$behavior compared to less neutron-rich neigbors. Self-consistent axially-deformed Hartree-Fock calculations, using a separable monopole interaction, of nuclei in the vicinity of ${ }^{190} \mathrm{~W}$ are performed to systematically examine the evolution of ground state quadrupole deformations. It is found that the neutron number $N=116$ causes a coexistence of oblate and prolate shapes, with a weak dependence on proton number, thereby hindering the development of these isotones as well-deformed rotors.
\end{abstract}

DOI: 10.1103/PhysRevC.72.047303

PACS number(s): 21.10.Pc, 21.60.Jz, 27.70.+q, 27.80.+w

It is well known that nuclei with one or more magic numbers are spherical in the ground state, and as one increases or decreases the number of protons or neutrons and moves to open shells, ground state deformation occurs. From a mean-field point of view, shape changes arise due to deformed single particle levels being energetically favored for incomplete $j$-shells. In the shell model, deformation occurs due to configuration mixing. In the interacting boson approximation (IBA), different nuclear shapes are described in terms of group theory, whereby axial prolate and oblate deformations, and spherical shapes can be described as realizations of different mathematical groups [1]. In the IBA, one can think of the control parameter related to the number of nucleons to be varied continuously, and can therefore refer to shape changes as a phase transition [2]. All these theoretical descriptions of nuclei, to the extent that they are correct, must be different, and complementary, ways of describing the same underlying physics.

Mapping the changes in the ground state shapes of nuclei is interesting because it helps illuminate these underlying theories. The shape of a nucleus also has a pronounced effect on its low-lying energy spectrum, and predictions of shape are valuable in interpreting data. For example, the ratio of energies of the first $4^{+}$excited state in an even-even nucleus to the energy of the first $2^{+}$state tends to 3.33 for a well-deformed rotational nucleus, and to 2.0 for a vibrational nucleus. Motivated by recent determination of the identities of low-lying states, and hence this ratio in ${ }^{190} \mathrm{~W}$ [3], and the prospects for forthcoming investigations of this mass region using radioactive isotope facilities, this paper studies, from a mean-field point of view, the evolution of ground state shape properties of nuclei in the region around ${ }^{190} \mathrm{~W}$. This part of the nuclear chart is particularly interesting, since it is, amongst heavy nuclei, the only place where one is several proton and neutron holes outside a doubly magic nucleus. Just below magic numbers, the energetics of low- $\Omega$ orbitals leads to small regions where oblate states may dominate at low spins, in contrast to the usual prolate dominance over most of the nuclear chart [4]. The experiment which reached ${ }^{190} \mathrm{~W}$ [3] is in the process of being followed up by further studies with projectile fragmentation reactions, allowing this oblate-dominant region to be studied in detail for the first time.
Figure 1 shows a summary of the lowest $4^{+}$to $2^{+}$energy ratio for nuclei in this region, where data are known $[3,5]$. The analysis of these empirical low-lying energy levels in ${ }^{190} \mathrm{~W}$ showed that whereas for the isotones $N=114$ and lighter the $E\left(4_{1}^{+}\right) / E\left(2_{1}^{+}\right)$ratio increases smoothly, tending towards 3.33 as $Z$ decreases from $Z=78$ to $Z=70$, for $N=116$ there is a decrease in this ratio between $Z=76(\mathrm{Os})$ and $Z=74(\mathrm{~W})$. The similarity of this result to the consequences of the breakdown of the $Z=64$ subshell gap for $N<78$ and $N>88$ suggested that the ${ }^{190} \mathrm{~W}$ result might be due to shell effects, particularly an already posited $Z=76$ subshell appearance [6], or equivalently, that a shape change occurs due to the shell effects [3].

Nuclei in this region, and specifically ${ }^{190} \mathrm{~W}$ have been the subject of previous theoretical investigation. The early IBA study of Duval and Barret [7] concentrated on the structure of lighter tungsten isotopes, and does not suggest an anomalous $E\left(4^{+}\right) / E\left(2^{+}\right)$ratio in ${ }^{190} \mathrm{~W}$. The pairing +quadrupole calculations of Kumar and Baranger [8] placed the shape change for osmium and platinum at lower neutron number, but did not extend tungsten calculations to $N=116$. Recent mean field based calculations of Naik et al. [9], in which relativistic and nonrelativistic calculations with angular momentum projections were performed, mapped out signatures of collective behavior in this region as a function of $N$ and $Z$. Though there were some differences in the results between the different models and parameters in their study, they did not conclude that ${ }^{190} \mathrm{~W}$ was different in nature to nearby nuclei. Woods-Saxon-based calculations [10] have suggested the prolate-oblate shape change takes place between $N=114$ and $N=116$, with similar calculations suggesting $N=118$ [11].

In the present work, an analysis is made of the systematics of nuclei in the region of ${ }^{190} \mathrm{~W}$ using a separable monopole interaction [12]. This interaction was designed to be amenable to the addition of correlations in perturbation theory, giving small corrections to the mean-field result. At the level of onebody observables it has been demonstrated that corrections beyond the mean field are particularly small [13]. This gives us some confidence that our particular mean-field approach may be able to give a qualitative description of the nuclei in question, if we concentrate on one-body observables such 


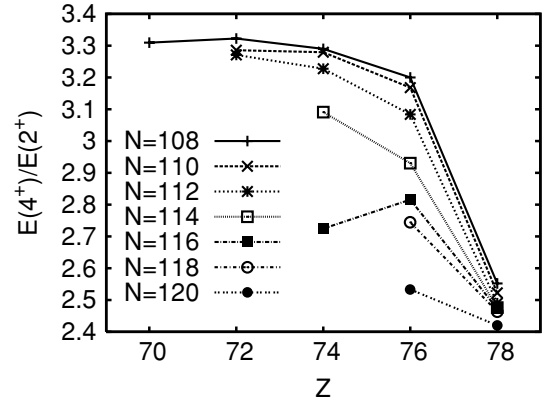

FIG. 1. Experimental $E\left(4^{+}\right) / E\left(2^{+}\right)$ratios for even-even isotones of $\mathrm{Yb}$ to Pt nuclei between $N=108$ and $N=120$. (Modified from Ref. [3]). as deformation. The interaction has also been used to study symmetric and asymmetric nuclear matter, and its equation of state has led to realistic models of neutron stars [14], giving credibility to applying it to nuclei as one moves away from stability to the neutron rich side.

Using the separable monopole interaction, Hartree-Fock mean-field calculations of $\mathrm{Yb}, \mathrm{Hf}, \mathrm{W}, \mathrm{Os}$, and Pt isotopes with neutron number ranging from 110 to 122 were performed using a constraint on the quadrupole deformation to map out the potential energy surface. The possibility of triaxial deformation was not allowed in the calculations. In this region, one may expect the nuclei to be rather soft in the gamma degree of freedom, and one should interpret the axially-symmetric calculations in that light. When discussing the experimental

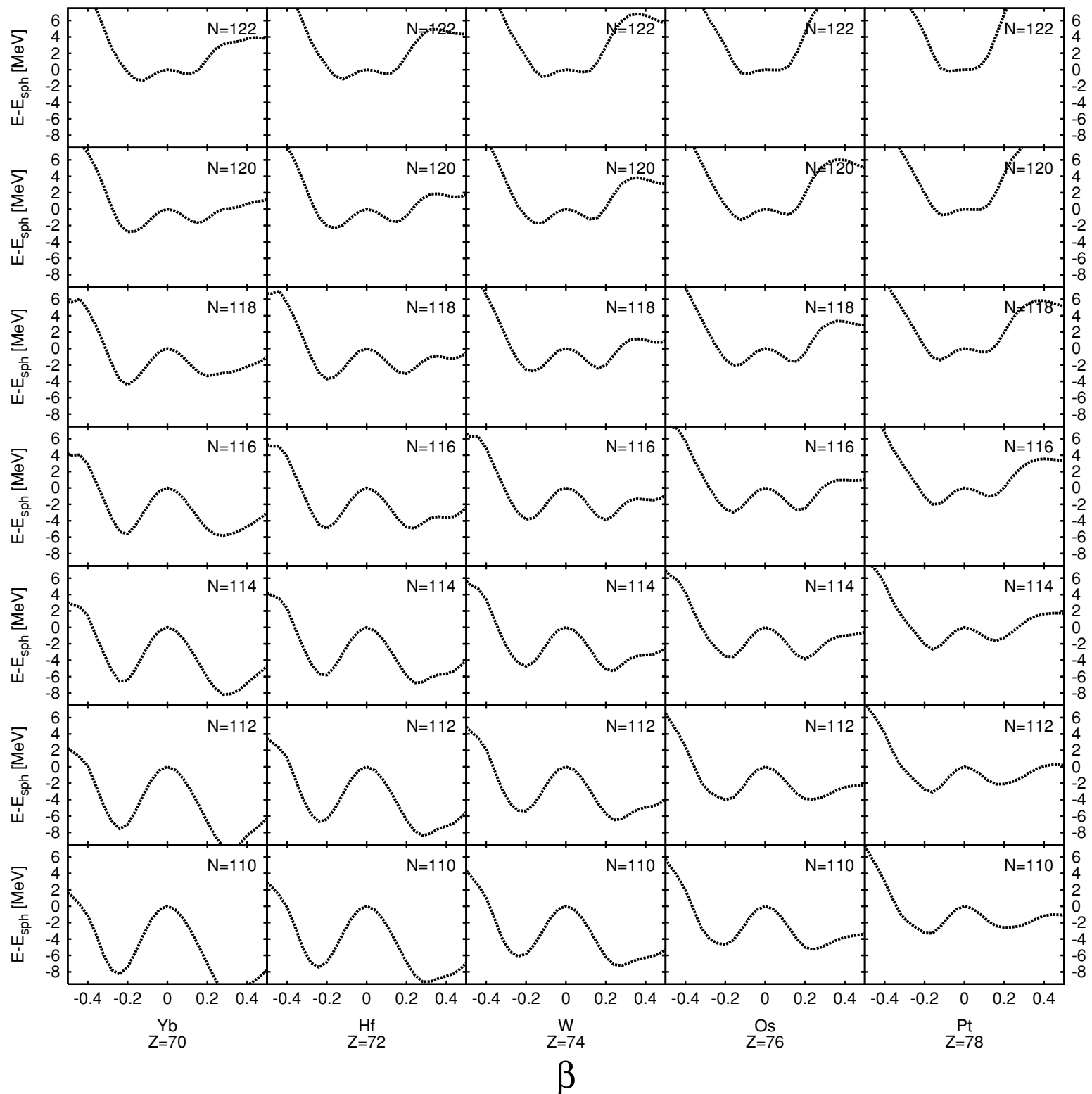

FIG. 2. Potential energy plots of nuclei with atomic number between $Z=70(\mathrm{Yb})$ and $78(\mathrm{Pt})$ and neutron number between $N=110$ and $N=122$. The binding energy of the spherical configuration is subtracted in each case to show the evolution of deformation energy as neutron number changes. 


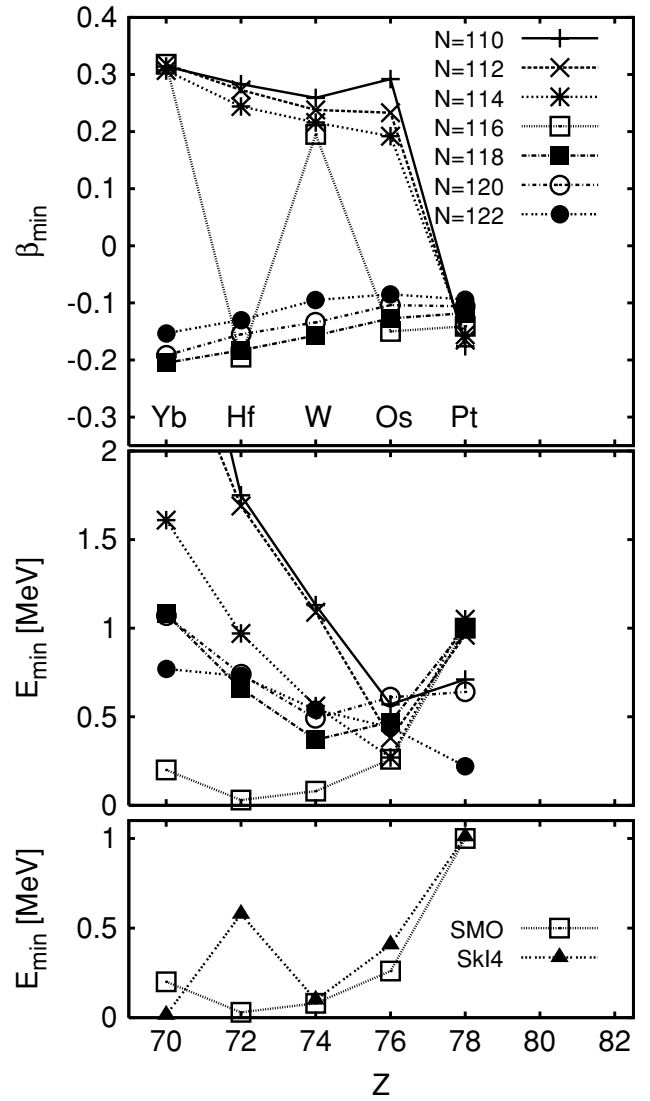

FIG. 3. Deformation at minima (upper panel) for isotones in the region of ${ }^{190} \mathrm{~W}$, the absolute difference between the minimum energy and the opposite shape minimum for the range of nuclides with the separable monopole interaction (central panel), and a comparison between the separable interaction (SMO) and a Skyrme parametrization (SkI4) for the case of $N=116$ (lower panel).

data on ${ }^{190} \mathrm{~W}$, a triaxial Woods-Saxon mean field calculation of the potential energy surface was performed [3], allowing for both $\beta$ and $\gamma$ degrees of freedom. In their case a barrier of the order a few hundred $\mathrm{keV}$ was found in the $\gamma$ direction, though this barrier was rather smaller than in the $\beta$ direction. We may consider in the following discussion that the softness in the $\gamma$ degree of freedom is comparable or greater than that in $\beta$, and qualitatively account for triaxial effects in the interpretation of any coexisting axial minima found.

The potential energies of the above nuclides as a funciton of $\beta$ deformation are shown in Fig. 2. One can see that as one approaches either the $N=126$, towards the top of Fig. 2, or the $Z=82$ shell closure, towards the right of Fig. 2 , the potential energy surfaces soften and the absolute $\beta$ values of the minima decrease, as expected. In the center of the plot is ${ }^{190} \mathrm{~W}$, which appears to have two minima of rather equal depth separated by a barrier of about $4 \mathrm{MeV}$. Assuming that this nucleus is indeed rather soft in the $\gamma$ direction, the equality of the depth of the oblate and prolate minima suggestes that ${ }^{190} \mathrm{~W}$ could be an ideal candidate for an $\mathrm{O}(6)$ nucleus, from an IBA point of view [2].

To partially account for the softness in the $\gamma$ direction, the constrained Hartree-Fock calculations which give rise to the potential energy surfaces are presented in a different way in Fig. 3. The upper part of the figure shows the deformation of the intrinsic ground state as predicted by the Hartree-Fock calculations. Following Ref. [3], isotonic lines are plotted for the series of even-even isotopes around tungsten. The suggestion from this plot is that for $Z=78$, the closeness to the $Z=82$ magic number is enough to ensure that all the Pt isotopes are oblate, but as one considers lower atomic numbers, away from the closed shell, the isotones split into those which have well-deformed prolate ground states $(N=110,112,114)$, and those whose neutron number is close enough to the $N=126$ magic number to keep the ground state oblate $(N=118,120,122)$. The $N=116$ isotones, appear to alternate between producing oblate and prolate ground states. The central panel of Fig. 3 helps interpret the meaning of these ground state deformations. It shows the difference in energies between the ground state, be it oblate or prolate, and the next minimum in the potential energy surface, which is of the opposite shape. It shows that as one decreases $Z$ away from the spherical $Z=82$ shell closure, the ground state deformation becomes increasingly rigid and well defined for most of the isotonic chains. The exception is $N=116$, in which the oblate and prolate minima continue to compete, as indicated by the small difference in energy between the two configurations. This leads one to conclude that the alternating $N=116$ line in the upper plot of Fig. 3 is due not to a definite alternating of shape, but to a lack of a definite rigid shape in the ground state of the $N=116$ isotones. The fact that the more well-defined shapes are separated by the $N=116$ line suggests that the oblate-prolate shape change in this region is a neutron-driven effect. In the lower part of Fig. 3, a comparison is made between the separable monopole force calculations for the $N=116$ isotones and a well-established Skyrme parametrization, SkI4 [15], to check that the presented results are not an anomaly of the choice of force. A good agreement is seen for the energy differences, with SkI4 also exhibiting a strong shape coexistence for all the $N=116$ isotones. A somewhat larger value of the energy difference between oblate and prolate minima is seen in ${ }^{188} \mathrm{Hf}$ in SkI4, yet it is still rather small, compared to the calculations for other hafnium isotopes, as seen in the central panel. The experimental determination of the low-lying spectrum of this isotope will be useful in differentiating between effective interactions. So far, only the ground state has been observed experimentally, following production in projectile fragmentation reactions [16].

The inclusion of ground state correlations is unlikely to qualitatively affect the results for those nuclei whose oblateprolate energy difference is large. However, it may change the staggering in the $N=116$ isotones, since the oblateprolate difference is so small, and this point should reinforce the above statement that, around ${ }^{190} \mathrm{~W}$, the $N=116$ isotones are not well-deformed rigid nuclei. The low $E\left(4^{+}\right) / E\left(2^{+}\right)$ ratio is then not surprising. The calculations performed with the separable monopole interaction, and presented here, further suggest that the effect will continue for ${ }^{188} \mathrm{Hf}$, and perhaps to lighter isotones.

In conclusion, constrained Hartree-Fock calculations for a series of even-even nuclei around ${ }^{190} \mathrm{~W}$ have been 
performed, showing that the $N=116$ isotones do not have a well-defined axially-deformed shape as $Z$ decreases around tungsten. The apparent anomaly in the systematics of the ratio of the first $4^{+}$and $2^{+}$energies in ${ }^{190} \mathrm{~W}$ is therefore explained by it not being a rigid axial rotor, unlike lighter tungsten isotopes.
This trend is expected to continue at least to the next even-even $N=116$ isotone, ${ }^{188} \mathrm{Hf}$.

This work was supported by the U.K. EPSRC and by U.S. DOE Grant No. DE-FG02-94ER40834. Zs.P. acknowledges financial support by the EPSRC under GR/A10789/01.
[1] J. Jolie and A. Linnemann, Phys. Rev. C 68, 031301(R) (2003).

[2] J. Jolie, R. F. Casten, P. von Brentano, and V. Werner, Phys. Rev. Lett. 87, 162501 (2001).

[3] Zs. Podolyák et al., Phys. Lett. B491, 225 (2000).

[4] N. Tajima and N. Suzuki, Phys. Rev. C 64, 037301 (2001).

[5] R. Firestone et al., Table of Isotopes, 8th ed. (Wiley and Sons, New York, 1996).

[6] H. Mach, Phys. Lett. B185, 20 (1987).

[7] P. D. Duval and B. R. Barrett, Phys. Rev. C 23, 492 (1981).

[8] K. Kumar and M. Baranger, Nucl. Phys. A110, 529 (1968).

[9] Z. Naik, B. K. Sharma, T. K. Jha, P. Arumugam, and S. K. Patra, Pramana 62, 827 (2004).
[10] W. Nazarewicz, M. A. Riley, and J. D. Garrett, Nucl. Phys. A512, 61 (1990).

[11] C. Wheldon et al., Phys. Rev. C 63, 011304(R) (2000).

[12] P. Stevenson, M. R. Strayer, and J. R. Stone, Phys. Rev. C 63, 054309 (2001).

[13] P. D. Stevenson, J. R. Stone, and M. R. Strayer, Phys. Lett. B545, 291 (2002).

[14] J. R. Stone, P. D. Stevenson, J. C. Miller, and M. R. Strayer, Phys. Rev. C 65, 064312 (2002).

[15] P.-G. Reinhard and H. Flocard, Nucl. Phys. A584, 467 (1995).

[16] B. Singh, Nucl. Data Sheets 95, 387 (2002). 